\title{
DNA Methylation Inhibition
}

National Cancer Institute

\section{Source}

National Cancer Institute. DNA Methylation Inhibition. NCI Thesaurus. Code C40575.

DNA Methylation Inhibition involves interference with, or restraint of, the process by which methyl groups are added to genomic DNA nucleotides. 